\section{Who's afraid of guidelines?}

Das Thema "Guidelines" wird gerne kontrovers diskutiert. Manche lehnen Guidelines etwas reflexartig $a b$, andere sind vom ihrem Nutzen überzeugt und fördern ihre Entwicklung, meist im Einklang mit den Idealen der Evidence-based Medicine. Bisher lagen aber keine zuverlässigen Angaben vor, ob und wie die Schweizer Ärzteschaft Guidelines akzeptiert bzw. weiterentwickeln möchte.

In dieser Ausgabe veröffentlichen wir zwei Arbeiten von Reto Obrist, welcher diesen Fragen in verdienstvoller Weise nachgegangen ist (zwei weitere Artikel folgen in der nächsten Nummer). Im Beitrag "Guidelines" [1] werden zunächst die wichtigsten Aspekte zum Thema beleuchtet. Allen, die den Einstieg in diese Thematik noch nicht recht finden konnten, sei die Lektüre des nuanciert und ausgewogen formulierten Textes empfohlen; er ist geeignet, während der noch im Aufbau befindlichen, von der FMH mitgetragenen "Guideline-Bewegung" möglichst viele an Bord des "think tanks" zu holen. Immerhin scheinen Guidelines in den Fachgesellschaften - dies zeigt die zweite Arbeit von Obrist [2] - eine bessere Akzeptanz zu finden, als herkömmlicherweise angenommen wird. Dies kann an der noch überschaubaren, aber unverkennbar zunehmenden Zahl erarbeiteter Guidelines abgelesen werden. Aber vor allem die Tatsache, dass die befragten Mitglieder der Fachgesellschaften die Einhaltung von Guidelines auf immerhin 60\% schätzen, ist ein ermutigender Hinweis, dass bisher ein vernünftiger Umgang mit dieser neuen Art der medizinischen Wissensvermittlung gefunden wurde.

Sind Guidelines denn wirklich etwas Neues? Welches Lehrbuch, welcher Fortbildungsartikel zielt letztlich nicht auch auf einen Zustand des «immer besser sein Wollens»? Ein Unterschied liegt vielleicht darin, dass Guidelines im Zusammenhang mit EBM und durch das Interesse nicht-ärztlicher Kreise eine besondere Dynamik erhalten haben. Vor allem aber unterscheiden sich Guidelines von Lehrbüchern und Fortbildungsartikel durch die an Wissenschaftlichkeit - genauer: wissenschaftliche Methodik - und Transparenz gestellten Ansprüche: systematische
Berücksichtigung und Wertung des Schrifttums, Ausarbeitung in einem sozialen, interessierte Kreise involvierenden Prozess, Offenlegung der finanziellen Verflechtungen und Interessenkonflikte und - last but not least - an klinische Resultate messbare Verhaltensänderungen der Adressaten. Vielleicht sind es gerade diese hohen Ansprüche - und die damit verbundenen Aufwendungen! -, welche vielerorts Skepsis bis Ablehnung gegenüber Guidelines hervorrufen. Nebenbei und etwas häretisch bemerkt, wäre es durchaus einmal lohnend zu prüfen, ob eine zu fünfstelligem Betrag erarbeitete, allen nur denkbaren Wünschen genügende Guideline tatsächlich zu signifikant besseren klinischen Resultaten führt als die prägnante Botschaft eines - gesponserten und/aber/ weil gewissenhaften - Opinion leaders zum gleichen Thema. Und selbst bei positivem Nachweis müsste noch eine Kosten-Nutzen-Analyse in eine abschliessende Übungskritik miteinbezogen werden. Es darf spekuliert werden!

Genügen rein utilitaristische Rechtfertigungsversuche, um die Akzeptanz von Guidelines zu fördern? Vielleicht muss zusätzlich deutlich gemacht werden, dass die Erarbeitung einer Guideline an sich schon einen faszinierenden Prozess darstellt. Faszinierend deshalb, weil kaum anderswo mit der gleichen Schärfe bewusst wird, dass Wissenschaft - im besten Fall - stets in einem sozialen Prozess erarbeitet wird und damit im Ergebnis auch ein soziales Gebilde bleibt. Wer nun Medizin als Wissenschaft versteht und weiterhin verstehen will - wenigstens teilweise, aber immerhin! -, müsste eigentlich erkennen, dass Guidelines eine wenn auch nicht exklusive so doch ideale Möglichkeit bieten, sich aktiv an diesem Wissenschaft erarbeitenden Prozess zu beteiligen, um ihn nicht dem Zufall - Zerfall? - zu überlassen. Wäre nicht alleine aufgrund dieser Erkenntnis schon genug Motivation gegeben, sich rational und konstruktiv mit dem Thema "Guidelines" weiterhin zu befassen?

Markus Trutmann

1 Obrist R. Guidelines. Was sie sollten und was sie tun. Schweiz Ärztezeitung 2001;82:1278-81.

2 Obrist R. Guidelines in den schweizerischen medizinischen Fachgesellschaften. Eine Bestandesaufnahme. Schweiz Ärztezeitung 2001;82:1282-5 